\title{
Working Within and Beyond the Cochrane Collaboration to Make Systematic Reviews More Useful to Healthcare Managers and Policy Makers
}

Travailler au sein de la Cochrane Collaboration et au delà de celle-ci pour rendre les examens systématiques plus utiles aux gestionnaires et aux décideurs du domaine des soins de santé

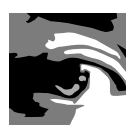

by JOHN N. LAVIS, MD, PHD

Member, Centre for Health Economics and Policy Analysis

Associate Professor, Department of Clinical Epidemiology and Biostatistics Associate Member, Department of Political Science McMaster University, Hamilton, Canada

HUW T.O. DAVIES, PHD

Director, Centre for Public Policy and Management

Professor, School of Management

University of St. Andrews, St. Andrews, Scotland

RUSSELL L. GRUEN, MB BS, PHD, FRACS

Fellow in Trauma Surgery and Critical Care, Harborview Medical Center and University of Washington, Seattle, USA

Associate Professor, Menzies School of Health Research, Darwin, Australia 
John N. Lavis, Huw T.O. Davies, Russell L. Gruen, Kieran Walshe and Cynthia M. Farquhar

KIERAN WALSHE, PHD

Director, Centre for Public Policy and Management

Professor of Health Policy and Management

Manchester Business School, Manchester, England

CYNTHIA M. FARQUHAR, MD, MPH, FRANZCOG

Professor, Department of Obstetrics and Gynaecology

University of Auckland, Auckland, New Zealand

\begin{abstract}
Participants in the Cochrane Collaboration conduct and periodically update systematic reviews that address the question,"What works?" for healthcare interventions. The Cochrane Library makes available quality-appraised systematic reviews that address this question. No coordinated effort has been undertaken to conduct and periodically update systematic reviews that address the other types of questions asked by healthcare managers and policy makers, to adapt existing reviews to highlight decision-relevant information (including the factors that may affect assessments of a review's local applicability) or to facilitate their retrieval through a "one-stop shopping" portal. Researchers interested in evaluating new methodological developments, health services and policy researchers interested in conducting and adapting systematic reviews, and research funders all have a role to play in making systematic reviews more useful for healthcare managers and policy makers.
\end{abstract}

\title{
Résumé
}

Les participants à la Cochrane Collaboration effectuent et mettent périodiquement à jour des examens systématiques qui abordent la question : "Qu'est-ce qui fonctionne? » pour les interventions en matière de santé. La Cochrane Library met, à la portée du public, des examens systématiques dont la qualité a été évaluée et qui traitent de cette question. Aucun effort coordonné na été entrepris pour effectuer et mettre périodiquement à jour des examens systématiques qui traitent des autres types de questions que posent les gestionnaires et les décideurs; pour adapter les examens existants afin de mettre en relief les données pertinentes pour la prise de décisions ( $y$ compris les facteurs susceptibles d'influencer les évaluations de l'applicabilité d'un examen à l'échelle locale); ou pour faciliter leur extraction par l'entremise d'un « guichet unique. » Les chercheurs désireux dévaluer les nouveaux développements méthodologiques, les chercheurs en politiques qui veulent réaliser et adapter des examens systématiques, ainsi que les bailleurs de fonds de travaux de recherche ont tous un rôle à jouer pour rendre les examens systématiques plus utiles aux gestionnaires et aux décideurs du domaine des soins de santé. 


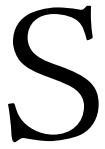

YSTEMATIC REVIEWS CAN INFORM HEALTHCARE MANAGEMENT AND POLICY making by providing research-based responses to important questions about health systems (Davies et al. 2000; Lavis et al. 2004). Systematic reviews offer four advantages to potential target audiences outside the research community, the first two of which apply primarily to reviews that address questions about "what works." First, the likelihood of being misled by research evidence is lower with a systematic review than with an individual study (Eggar et al. 2001). Second, confidence in what can be expected from an intervention is higher with a systematic review than with an individual study (Eggar et al. 2001). Third, drawing on an existing systematic review constitutes a more efficient use of time because the research literature has already been identified, selected, appraised and synthesized in a systematic and transparent way (Lavis et al. 2005). Fourth, a systematic review can be more constructively contested than an individual study because debates can focus on appraisal and synthesis rather than on the reasons that one study was identified and selected over others (Lavis et al. 2005).

The Cochrane Collaboration is an international, not-for-profit and independent organization, dedicated to making up-to-date, accurate information about the effects of healthcare interventions readily available worldwide by promoting the search for evidence and producing and disseminating systematic reviews. The Cochrane Library provides one-stop shopping for quality-appraised reviews that address the question "What works?" - both those reviews produced according to the quality standards of the Cochrane Collaboration and those that have been quality-appraised by two independent raters. (The Cochrane Library also provides one-stop shopping for health technology assessments, which typically build on systematic reviews, and economic evaluations.)

The Cochrane Collaboration's Effective Practice and Organization of Care (EPOC) Review Group has as its major focus the promotion of systematic reviews of health system interventions (McAuley et al. 2003). An EPOC review draws on randomized controlled trials or (in their absence) controlled before/after studies and interrupted time-series studies to address a question about the effectiveness of an intervention (i.e.,"What works?"). EPOC faces challenges, however, in ensuring that reviews address questions relevant to healthcare management and policy making, developing methods and quality standards to assess complex health system interventions, highlighting factors that may influence the local applicability of reviews and adapting the presentation of reviews to enhance their usefulness for managers and policy makers.

No coordinated effort akin to the Cochrane Collaboration has been undertaken to address questions other than "What works?", and no "one-stop shopping" portal akin to the Cochrane Library has been developed to make available quality-appraised 
reviews that address a broader array of questions. Healthcare managers and policy makers are interested in the most effective solutions to the most burdensome health problems, the most effective ways to fit these solutions into complex health systems and the most effective ways to bring about desired changes in health systems (Lavis et al. 2004). But in addition to asking questions about effectiveness (does changing $X$ change $Y$ ?), they also ask questions about cost-effectiveness (is $X_{1}$ more cost-effective than $X_{2}$ in achieving a one-unit change in $Y$ ?), relationships (is $X$ associated with $Y$ ?), mechanisms (how are $X$ and $Y$ linked, or why does changing $X$ change $Y$ ?) and meanings (how have $\mathrm{X}$ or $\mathrm{Y}$ been viewed or experienced?). In recent years a variety of new approaches have been developed to conduct and update systematic reviews that address this broader array of questions.

In this paper we outline some ways in which the production and updating of systematic reviews (i.e., the future flow of systematic reviews), the adaptation of the global stock of systematic reviews and the development of improved retrieval mechanisms for systematic reviews could enhance the usefulness of systematic reviews for healthcare managers and policy makers. In so doing we highlight how health services and policy researchers can work both within the Cochrane Collaboration to address questions about "what works" and beyond it to address other questions. We envision a future in which all health services and policy researchers register their reviews (as do Cochrane reviewers) to avoid unnecessary duplication and most regularly update at least one systematic review. We also envision a future in which research funders support production, adaptation and retrieval processes to ensure that systematic reviews are available when healthcare managers and policy makers need them to inform their decision-making.

\section{Conducting and Updating Systematic Reviews}

Of the five elements of a systematic review - (1) an explicit question, (2) an explicit description of the search strategy, (3) an explicit statement about what types of research evidence were included and excluded, (4) a critical examination of the quality of the studies included in the review and (5) a critical and transparent process of interpretation of the findings of the studies included in the review - we focus particularly on posing questions (element 1), selecting studies (element 3 ) and synthesizing studies (element 5), as well as on the role of healthcare managers and policy makers in these three steps.

We begin with involving healthcare managers and policy makers in the systematic review because their inclusion has the potential to influence many elements of the process. We offer three reasons for augmenting the stock of investigator-driven systematic reviews with reviews that involve healthcare managers and policy makers. First, a systematic review of the factors that influenced the use of research evidence 
in healthcare policy making identified that individual-level interactions between researchers and healthcare policy makers increased the prospects for research use in policy making (Lavis et al. 2005). Second, an analysis of websites of research funders, producers/purveyors of research and journals that include healthcare managers and policy makers among their target audiences found that such linkage and exchange processes are rare (Lavis et al. 2005). Third, involving managers and policy makers in the systematic review could enhance the public accountability of researchers when they derive take-home messages from research, which is a type of accountability that has been noticeably lacking (Black 2001).

We now turn to the first element of a systematic review - an explicit question. As we have already pointed out, healthcare managers and policy makers ask questions about the most effective solutions to the most burdensome health problems, the most effective ways to fit these solutions into complex health systems or, more generally, to design health systems (i.e., governance, financial and delivery arrangements) and the most effective ways to bring about desired changes in health systems. While Cochrane's EPOC Review Group is focused in part on the effectiveness of such governance, financial and delivery arrangements, the scale of its effort does not yet match the scale (or complexity) of the task at hand. Moreover, while the EPOC Review Group is also focused in part on the most effective ways to bring about desired changes in health systems, its efforts need to be expanded beyond interventions targeted at health professionals to include change-management strategies at the level of organizations.

Healthcare managers and policy makers also ask questions about the cost-effectiveness of alternative approaches to achieving particular outcomes, relationships between factors and outcomes, mechanisms through which factors may affect outcomes and the meanings ascribed to particular factors and outcomes. In recent years new approaches have been developed to conduct and update systematic reviews that address this broader array of questions (Dixon-Woods et al. 2005; Mays et al. 2005). Most involve relatively minor alterations to established approaches, but one is substantively different in that it takes a more iterative approach to the development of the question as the systematic review progresses (Pawson et al. 2005). The arguments in favour of allowing the question to be refined and revised are that this approach is more likely to yield new ways of thinking and, when informed by interactions with healthcare managers and policy makers, is more likely to yield reviews relevant to the decisions they face. The arguments against allowing the question to change are that this approach requires either a great deal of resources or "cutting corners" in subsequent steps, and that it introduces bias into what would otherwise be an approach that strives to minimize bias.

We now turn to the third element of a systematic review - an explicit statement about what types of research evidence were included and excluded. Here we again highlight the one substantive change that has been advocated by some of those who 
produce systematic reviews for healthcare managers and policy makers: drawing a purposive sample of studies for review rather than reviewing all eligible studies. This proposal often goes hand in hand with the proposal to allow the question to change as the systematic review progresses, in part because drawing a sample of studies reduces the resources required for an iterative approach. Drawing a purposive sample of studies would also be consistent with the qualitative methods used in some approaches to synthesizing studies. The arguments against purposive sampling are that it could introduce bias and, in the long run, reduce the pressure to improve the retrievability of health services and policy research.

Finally, we turn to the fifth element of a systematic review - a critical and transparent process of interpretation of the findings of the studies included. The new approaches that have been developed to conduct and update systematic reviews that address the broader array of questions asked by healthcare managers and policy makers often differ most profoundly in how research findings are synthesized (DixonWoods et al. 2005). The approaches range from techniques that are largely qualitative and interpretive (e.g., thematic analysis) to those that are largely quantitative and integrative (e.g., Bayesian meta-analysis). A recent review of these approaches concluded with a call for their further development and refinement in coordinated and wellevaluated ways (Dixon-Woods et al. 2005).

\section{Adapting Systematic Reviews}

Even if the future flow of systematic reviews were modified in ways that enhance their relevance to healthcare managers and policy makers, substantial efforts would still be needed to adapt the global stock of reviews in ways that enhance their usefulness. Two potential adaptations involve changes to the types of information profiled in a systematic review. First, information about the harms (or risks) and costs of interventions (not just the benefits), the uncertainty associated with estimates and any differential effects by subgroup would be needed in order to provide healthcare managers and policy makers with decision-relevant information. Second, information about the contextual factors that may affect a review's local applicability would be needed in order for managers and policy makers to decide whether to give serious consideration to the decision-relevant information. The other potential adaptation involves developing user-friendly "front ends" for reviews that would allow rapid scanning for relevance and then graded entry to highly relevant reviews.

Providing three types of decision-relevant information - harms (or risks) and costs (not just benefits), uncertainty and differential effects by subgroup - was universally supported as a way to enhance the usefulness of systematic reviews by the healthcare managers and policy makers who were interviewed about these possibilities (Lavis et al. 2005). Highlighting the uncertainty associated with estimates would be 
relatively straightforward. But providing information about harms (or risks) would require greater emphasis on examining in primary studies the harms (or risks) associated with interventions (GRADE Working Group 2004). Providing information about the costs of interventions, not just the benefits, would require additional efforts to identify such costs as well as a broader consideration of economic issues in systematic reviews, a topic being examined by the Campbell and Cochrane Economics Methods Group (C\&CEMG 2005). Moreover, describing any differential effects by subgroup would need to be approached with caution, given prevailing concerns about subgroup analyses (Oxman and Guyatt 1992).

Providing information about the contextual factors that may affect a review's local applicability is perhaps even more important and challenging. Commonalities in human biology mean that a prescription drug will often work the same way in different populations. Differences in health systems mean that an intervention that works in one organization or jurisdiction may not work the same way in another, and systematic reviews may not contain studies that were conducted in a healthcare manager's organization or a policy maker's jurisdiction. One approach to helping managers and policy makers decide whether to give serious consideration to a systematic review is to highlight features of the intervention and the contexts in which it was employed that would influence assessments of the review's local applicability. Such features may include the relative importance of the health problem, relevance of outcome measures, practicality of the intervention, appropriateness of the intervention and its cost-effectiveness (Gruen et al. 2005).

A second approach to assisting managers and policy makers with assessments of the local applicability of a systematic review is to equip them with a tool to conduct such assessments (Lavis et al. 2004). The one existing tool includes four questions: (1) Could it work, or are there important differences in the structural elements of health systems that mean an intervention could not work in the same way as in the jurisdictions where the research was done? (2) Will it work, or are there important differences in the perspectives and influence of those health system stakeholders who have the political resources to influence decisions that mean an intervention will not be accepted or taken up in the same way, and does the health system face other challenges that substantially alter the potential benefits and harms (or risks) of the intervention? (3) What would it take to make it work, or can power dynamics and on-the-ground realities and constraints be changed in the short to medium term, and what are the prospects for making this happen? (4) Is it worth it or is the balance of benefits and harms (or risks) classifiable as net benefits, trade-offs, uncertain trade-offs or no net benefits, and are the incremental health benefits from incorporating the intervention among the mix of interventions provided worth the incremental costs?

Developing user-friendly "front ends" for reviews that allow rapid scanning for 
relevance and then graded entry constitute a third and very different type of adaptation process. One example of such a format is one page of take-home messages, a three-page executive summary that summarizes the full report, and a 25-page report, as well as a longer technical report, if necessary (Canadian Health Services Research Foundation 2001). Interviews with healthcare managers and policy makers suggest that presenting systematic reviews using something like a 1:3:25 format is preferred over current approaches. However, an analysis of websites suggests that reports using a graded-entry format are rare (Lavis et al.2005). Presumably, either the one- or three-page summary should follow a structured format. Structured abstracts are an innovation developed by those conducting clinical research (Haynes et al. 1990).

\section{Improving Retrieval Mechanisms for Systematic Reviews}

Even if the global stock and future flow of systematic reviews were modified in ways that enhance their relevance and usefulness to healthcare managers and policy makers, substantial efforts would still be needed to improve retrieval mechanisms. For systematic reviews to be helpful, managers and policy makers need to be able to access them when they need them. Three retrieval mechanisms are commonly used: (1) searching the Cochrane Library, (2) using the systematic review option in a PubMed clinical query (National Center for Biotechnology Information 2005) and (3) copying and pasting the best available search strategies (bmj.com 2005; Montori et al. 2004) into a PubMed query. These mechanisms have not yet been tested for systematic reviews of health services and policy research, for systematic reviews that address questions other than "What works?" or for databases other than Medline.

More importantly for healthcare managers and policy makers, the user-friendly "front ends" of systematic reviews could be made available through an online database that could be searched using keywords that make sense to managers and policy makers and that is linked to the full reviews when they are available through other quality-appraised sources, such as the Cochrane Library. The Health Evidence Network (WHO Regional Office for Europe 2005) provides a database targeted at healthcare policy makers; however, the evidence summaries are not always based on systematic reviews.

\section{Towards Shared Ground and Further Debate}

There is a great deal of shared ground in the perspectives of those advocating for an increased focus on systematic reviews as a way to provide research-based responses to important questions about health systems. For example, there is widespread agreement that the reviews should collectively (not necessarily individually) address a variety of questions relevant to healthcare managers and policy makers (including 
"What works?"). It is also generally agreed that methods should be systematic, transparent and appropriate to the question(s) asked; that new methods should be subject to evaluation (e.g., allowing the question to change once the review has been started, selecting a purposive sample of studies rather than all eligible studies and using different approaches to synthesizing eligible studies); and that the resulting products should be adapted to the needs of managers and policy makers (Sheldon 2005).

However, there are also some important differences of opinion (Lomas 2005). Some would argue that the Cochrane Collaboration's highly specified and routinized methodologies are appropriate to questions of "what works" for healthcare interventions such as drugs and procedures, but are likely to work less well and provide fewer useful insights when used to tackle a broader range of questions concerning complex organizational and policy interventions. But there are many areas of cross-over between these two research domains. For example, many social scientists also ask questions about "what works" - witness the Campbell Collaboration, which is focused on social, behavioural and educational interventions (rather than healthcare interventions) and the many social scientists working within the Cochrane Collaboration itself. In clinical research, too, there is a long tradition of examining mediating and moderating variables (including context). We would argue that the important point is that those who want to see healthcare managers and policy makers make better use of research in their decision-making should aim to learn from the considerable experience and accumulated expertise of the Cochrane Collaboration, while recognizing that its methods and approaches may need to be adapted and revised. These and other differences in perspective should be subject to further debate.

We summarize in Table 1 some dimensions of that debate where we believe that legitimate and important differing perspectives exist. In some cases we might expect a greater consensus to emerge, as experience of conducting systematic reviews on health system interventions accumulates; in other areas, the tensions will resolve differently contingent on the managerial or policy questions being asked. Health services and policy researchers could learn the hard way how best to conduct systematic reviews and not benefit from the experience of those who have grappled with similar challenges in other methodological and disciplinary domains, but few of us would argue that such an approach would be either sensible or a justifiable use of scarce research resources. Participants in the Cochrane Collaboration are likely to welcome and benefit themselves from the opportunity to tackle the challenges unique to producing and regularly updating systematic reviews for healthcare managers and policy makers.

\section{Implications for Researchers and Research Funders}

As the health services and policy research community begins to pay serious atten- 
TABLE 1. Some differing perspectives about producing and adapting a systematic review

ISSUE

\section{Developing a partnership for producing and adapting a systematic review}

\section{Conducting a systematic review}

Adapting a systematic review

\begin{abstract}
PERSPECTIVES
Should we engage with managers and policy makers at the start and end of the review process to set the question and interpret the findings, but let the methodological expertise of the researchers lead the intervening process; or, should we aim for a more iterative and continuing engagement throughout the review?
\end{abstract}

Should we address a focused question where we can do a good review, but risk its not speaking to many of the issues that managers and policy makers want it to tackle; or, should we tackle a broad question that is highly relevant but involves considerable methodological challenges?

Should we aim for a review process that is highly specified, routinized, methodologically sound and transparent, but which might be difficult to adapt to a broad question and heterogeneous literatures; or, should we have a more flexible and adaptable review process that can be tailored to fit the question, but risk being less robust, demonstrably rigorous and transparent?

Should we develop a review process that contributes to a global stock of systematic reviews on which all managers and policy makers can draw and that highlights information that can inform assessments of local applicability and develop (or leave to others to develop) a separate local adaptation process; or, should we combine production and local adaptation processes by incorporating both research evidence and information about managers' and policy makers' experiences and assessments of their local context?

tion to systematic reviews, now is the time for researchers who are interested in the methodology of systematic reviews or knowledge transfer and exchange to address a number of key issues in the production and adaptation of systematic reviews:

- evaluating alternative approaches to involving healthcare managers and policy makers in the systematic review process;

- evaluating alternative approaches to addressing the different types of questions asked by healthcare managers and policy makers, with a particular focus on such 
issues as the trade-offs involved in allowing the question to change as the systematic review progresses, drawing a purposive sample of studies for inclusion rather than reviewing all eligible studies and using different approaches for synthesizing research findings;

- evaluating alternative approaches to providing information about the contextual factors that may affect a review's local applicability;

- evaluating alternative approaches to developing user-friendly "front ends" for reviews, with a particular focus on the optimal structured format for these "front ends"; and

+ evaluating alternative approaches for retrieving systematic reviews of health services and policy research and systematic reviews for questions other than "What works?"

Much of this research could be conducted in conjunction with the Cochrane Collaboration. In pursuing this research agenda, care will need to be taken to identify both similarities and differences between healthcare managers and policy makers. For the purposes of this paper, we have considered them together; however, sometimes their differences may warrant a differentiated approach.

Health services and policy researchers who are interested in conducting and adapting systematic reviews for healthcare managers and policy makers can proceed with a number of key activities:

- involving healthcare managers and policy makers in the systematic review;

- working with Cochrane's EPOC Review Group to increase the scale of its efforts devoted to systematic reviews of the effects of governance, financial and delivery arrangements;

- working with Cochranés EPOC Review Group to expand the scope of their efforts devoted to systematic reviews of the effects of interventions to bring about change in health systems (i.e., include change-management strategies at the level of organizations, not just interventions targeted at health professionals);

- providing decision-relevant information in systematic reviews, with a particular focus on information about the harms (or risks) and costs of interventions (not just the benefits), the uncertainty associated with estimates and any differential effects by subgroup;

- developing something akin to the Cochrane Collaboration for questions other than "What works?"; and

- providing information about the contextual factors that may affect a review's local applicability.

Research funders could support the activities of researchers who are evaluating 
new methodological developments and health services and policy researchers who are conducting and adapting systematic reviews. The latter may require substantial investments in regularly undertaking priority-setting processes to identify emerging questions that could be addressed with systematic reviews (Lomas et al. 2003), the commissioning of "scoping" reviews to identify what types of full systematic reviews are warranted to address priority questions, and the training of health services and policy researchers to conduct and adapt systematic reviews. A single research funder, or a consortium of research funders, could also play a role in improving the retrievability of health services and policy research (randomized, controlled trials did not become easy to identify in Medline by chance alone) and in making available the user-friendly "front ends" of systematic reviews through an online database. For research funders who take seriously their role to make research more useful to healthcare managers and policy makers, systematic reviews offer tremendous opportunities.

\section{ACKNOWLEDGMENTS}

This paper was first prepared as a background paper for the first Harkness Fellows in Health Care Policy Alumni Conference, which was co-sponsored by the Commonwealth Fund, the Nuffield Trust and the Health Foundation, and was held at the Pennyhill Park Conference Centre, Bagshot, England, July 17-19, 2005. John Lavis receives salary support as the Canada Research Chair in Knowledge Transfer and Uptake.

Correspondence may be directed to: John N. Lavis, McMaster University, HSC-2D3, 1200 Main St. West, Hamilton, ON, Canada L8N 3Z5; tel.: +1-905-525-9140 (ext. 22907); fax: +1-905529-5742; email: lavisj@mcmaster.ca; Web: www.researchtopolicy.ca.

\section{REFERENCES}

Black, N. 2001. “Public Accountability: One Rule for Practitioners, One for Scientists?” Journal of Health Services Research and Policy 6(3): 130-132.

bmj.com. 2005."E-Table 1. PubMed Translation of Ovid Search Strategies to Retrieve Systematic Reviews from MEDLINE." Retrieved December 6, 2005. <http://bmj.bmjjournals.com/cgi/ content/full/bmj.38336.804167.47/DC1>.

Campbell and Cochrane Economics Methods Group (C\&CEMG). 2005. Retrieved December 6, 2005. <http://www.med.uea.ac.uk/research/research_econ/cochrane/cochrane_home.htm>.

Canadian Health Services Research Foundation. 2001. "Reader-Friendly Writing - 1:3:25." Retrieved December 6, 2005. <http://www.chsrf.ca/knowledge_transfer/pdf/cn-1325_e.pdf>. Davies, H.T.O., S.M. Nutley and P.C. Smith. 2000. What Works? Evidence-Based Policy and Practice in Public Services. Bristol, United Kingdom: The Policy Press.

Dixon-Woods, M., S. Agarwal, D. Jones, B. Young and A. Sutton. 2005. “Synthesising Qualitative and Quantitative Evidence: A Review of Possible Methods." Journal of Health Services Research and Policy 10(1): 45-53. 
Working Within and Beyond the Cochrane Collaboration to Make Systematic Reviews More Useful to Healthcare Managers and Policy Makers

Egger, M., G.D. Smith and K. O'Rourke. 2001."Rationale, Potentials, and Promise of Systematic Reviews." In M. Egger, G.D. Smith and D.G. Altman, eds. Systematic Reviews in Health Care: Meta-Analysis in Context (2nd ed.). London: BMJ Books.

GRADE Working Group. 2004. "Grading Quality of Evidence and Strength of Recommendations." British Medical Journal 328: 1490-1494.

Gruen, R.L., P.S. Morris, E.L. McDonald and R.S. Bailie. 2005. "Making Systematic Reviews More Useful for Policy-Makers." Bulletin of the World Health Organization 83(6): 480-481.

Haynes, R.B., C.D. Mulrow, E.J. Huth, D.G. Altman and M.J. Gardner. 1990. "More Informative Abstracts Revisited." Annals of Internal Medicine 113(1): 69-76.

Lavis, J.N., H.T.O. Davies, A.D. Oxman, J.-L. Denis, K. Golden-Biddle and E. Ferlie. 2005.

"Towards Systematic Reviews That Inform Health Care Management and Policy-Making." Journal of Health Services Research and Policy 10 (Supplement 1): S1:35-S1:48.

Lavis, J.N., F.B. Posada, A. Haines and E. Osei. 2004. “Use of Research to Inform Public Policymaking." The Lancet 364(9445): 1615-1621.

Lomas, J. 2005. “Using Research to Inform Healthcare Managers' and Policy Makers' Questions: From Summative to Interpretive Synthesis." Healthcare Policy 1(1): 55-71.

Lomas, J., N. Fulop, D. Gagnon and P. Allen. 2003. “On Being a Good Listener: Setting Priorities for Applied Health Services Research." Milbank Quarterly 81(3): 363-388.

Mays, N., C. Pope and J. Popay. 2005. "Systematically Reviewing Qualitative and Quantitative Evidence to Inform Management and Policy-Making in the Health Field." Journal of Health Services Research and Policy 10 (Supplement 1): S1:6-S1:10.

McAuley, L., J.M. Grimshaw and M. Zwarenstein. 2003. "Scope of EPOC Is Clarified." British Medical Journal 327: 106.

Montori, V.M., N.L. Wilczynski, D. Morgon and R.B. Haynes. 2004."Optimal Search Strategies for Retrieving Systematic Reviews from Medline: Analytical Survey" British Medical Journal 330: $1-6$.

National Center for Biotechnology Information. 2005. "PubMed Clinical Queries." Retrieved December 6, 2005. <http://www.ncbi.nlm.nih.gov/entrez/query/static/clinical.html>.

Oxman, A.D. and G.H. Guyatt. 1992. “A Consumers' Guide to Subgroup Analyses." Annals of Internal Medicine 116: 78-84.

Pawson, R., T. Greenhalgh, G. Harvey and K. Walshe. 2005. "Realist Review - A New Method of Systematic Review Designed for Complex Policy Interventions." Journal of Health Services Research and Policy 10 (Supplement 1): S1:21-S1:34.

Sheldon, T.A. 2005. "Making Evidence Synthesis More Useful for Management and PolicyMaking." Journal of Health Services Research and Policy 10 (Supplement 1): S1:1-S1:5.

World Health Organization Regional Office for Europe. 2005. "Health Evidence Network (HEN)." Retrieved December 6, 2005. <http://www.who.dk/hen>. 